\title{
Beyond the Experience: Detection of Metamemorial
}

\author{
Regularities \\ Marie Geurten ${ }^{1}$, Sylvie Willems ${ }^{2}$, \& Thierry Meulemans ${ }^{1}$ \\ ${ }^{1}$ Department of Psychology: Cognition and Behavior, Neuropsychology Unit, University of \\ Liège, Belgium \\ ${ }^{2}$ Psychological and Speech Therapy Consultation Center (CPLU)
}

\author{
Corresponding author \\ Marie Geurten \\ Department of Psychology: Cognition and Behavior \\ Neuropsychology Unit \\ University of Liège \\ Boulevard du Rectorat - B33 \\ 4000 Liège \\ BELGIUM \\ mgeurten@ulg.ac.be \\ Phone: +3243665943
}




\begin{abstract}
We examined the mechanisms involved in the development of the easily learned, easily remembered (ELER) heuristic in three groups of young children (4-5 years, 6-7 years, and 8-9 years). A trial-to-acquisition procedure was used to evaluate how much these children's judgment of learning depended on the ELER heuristic. Moreover, a new experimental paradigm, composed of six phases-a pretest, four training phases, and a posttest-was employed to implicitly influence the validity of the ELER association that underlies this metacognitive rule. Results revealed that the ELER heuristic develops early (4-5 years), but its use is reduced after implicit training. Furthermore, executive monitoring was found to account for the smaller changes observed in older children (8-9 years) after training. From a developmental perspective, these findings present a coherent picture of children's learning of metacognitive heuristics, wherein early automatic and implicit learning is later followed by effortful control.
\end{abstract}

Keywords: Implicit learning, heuristics, metamemory, judgment, children 


\section{Introduction}

It is commonly hypothesized that metamemory knowledge develops throughout childhood on the basis of day-to-day experience (Jacoby, Kelley, \& Dywan, 1989; Olds \& Westerman, 2012). Over the past decade, several studies have examined the nature of the mechanisms that underlie this experiential development (Grammer, Purtell, Coffman, \& Ornstein, 2011; Hodzik \& Lemaire, 2011). To date, these studies have mostly focused on explicit metacognitive knowledge - that is, late-onset knowledge that people can verbalize and purposely put into practice when they are confronted with tricky memory situations-and have generally demonstrated that high-level cognitive functions are required to acquire knowledge about memory functioning (Grammer et al., 2011).

In reality, however, metamemory is not fully explicit (Lyons \& Ghetti, 2013; Paulus, Proust, \& Sodian, 2013). Many metacognitive theories merely guide memory decisions at the border of awareness. For example, we use the ease with which memories of positive events come to mind as a cue to determine whether we had a good time (availability heuristic; Tversky \& Kahneman, 1973); we judge the familiarity of names on the basis of the fluency with which we process them (fluency heuristic; Johnston, Hawley, \& Elliott, 1991); and we are inclined to suppose that easily learned information is more likely to be remembered (memorizing-effort heuristic; Koriat, 2008). These naïve theories and the underlying heuristic rules are rarely, if ever, verbalized or deliberately employed, but their effects on memory performance are equal to those of explicit strategies (Dodson \& Schacter, 2001; Ghetti, 2008; Howe, 2008; Meeks, Knight, Brewer, Cook, \& Marsh, 2014). However, although researchers studying adult metacognition have long placed a heavy emphasis on the study of these heuristic rules, research on metacognition in children has only recently started to pay greater attention to the contribution made by these heuristic-based judgments (e.g., Koriat, Ackerman, Lockl, \& Schneider, 2009a; Lock1 \& Schneider, 2002; Roebers, von der Linden, Schneider, \& Howie, 2007). Generally, the results of these studies indicate that memory decisions are already based on some heuristics (e.g., the memorizing-effort or availability heuristic) by the age of 7 to 8 years. Thus far, the question of whether the same is true of younger children has mostly remained unexplored. Furthermore, to our knowledge, no previous study has examined the processes that explain the development of these metacognitive rules. The present study is an attempt to fill these gaps.

Recently, several studies have demonstrated that metacognitive heuristics in adults are more malleable and sensitive to environmental specificity than had previously been thought (e.g., Olds \& Westerman, 2012; Unkelbach, 2006; Winkielman \& Schwarz, 2001). Unkelbach (2006, 2007), for instance, has established that the fluency heuristic can be reversed when participants are trained to associate the subjective feeling of difficulty with familiarity, which seems to indicate that people are able to temporarily modify the metacognitive content of their heuristics when they perceive new environmental constants. For example, in one of these experiments (Unkelbach, 2006, Experiment 1), participants made recognition judgments in which processing fluency was manipulated by color contrast. This test was preceded by a learning phase specifically constructed to thwart the natural fluency-familiarity relationship: previously learned (i.e., familiar) stimuli were systematically presented in low contrast (i.e., dysfluent condition) and new (i.e., unfamiliar) stimuli were systematically presented in high contrast (fluent condition). The results revealed that participants were quickly able to learn that fluent processing indicated that the stimulus was new and to use this novel information to guide their memory decision during the recognition test. This leads us to hypothesize that children may learn the knowledge underlying metacognitive heuristics by means of a well-established implicit process 
involving the detection of environmental regularities. In non-metacognitive contexts, this aptitude appears early in development (Meulemans, Van der Linden, \& Perruchet, 1998), sometimes as early as the age of 1 (Gomez \& Gerken, 1999). We therefore hypothesized that children exposed to repeated-but not explicitly mentioned-memory contingencies should be able to detect the underlying rule and then use it as a guide to predict their future recall.

For this purpose, we chose to base our experiment on the easily learned, easily remembered (ELER) heuristic, whereby the easier it feels to learn new information, the better people judge that their ability to recall it in the future will be (Koriat, 2008; Koriat, Nussinson, \& Ackerman, 2014; Paulus, Tsalas, Proust, \& Sodian, 2014; Undorf \& Erdfelder, 2011). This heuristic was initially examined by Koriat (2008; see also Koriat, Ma'ayan, \& Nussinson, 2006). In that study, participants were required to encode a list of paired-associates using a classical trial-to-acquisition procedure (i.e., a study-test loop procedure in which pairs that participants fail to recall are presented for re-study until perfect recall is achieved; Bahrick \& Phelps, 1987) followed by a judgment of learning (JOL) solicitation. The results indicated that participants' JOLs for items were negatively correlated with the number of trials needed to achieve perfect recall of those items at study. In other words, the more trials were needed to learn an item, the lower was participants' prediction of their future recall of the item. Moreover, this heuristic offers the double advantage of running counter to the observation that memory performance actually improves with repeated practice-which explains why children often have no explicit knowledge of the heuristic (Koriat \& Ackerman, 2010; Koriat, Ackerman, Lockl, \& Schneider, 2009b) - and of having demonstrated sensitivity to experimental manipulation (Koriat, Ackerman, Adiv, Lockl, \& Schneider, 2014; Miele, Finn, \& Molden, 2011).

For these reasons, we decided to use this heuristic to determine whether children are able to learn a new piece of metacognitive knowledge by detecting environmental and phenomenological regularities. Specifically, since we are not aware of any studies on the ELER heuristic before the age of 7 (Koriat et al., 2009a), we cannot rule out the possibility that this particular heuristic is at least partially established at a very young age. And in fact, if the implicit detection of environmental regularities truly underlies the learning of metacognitive heuristics, it is highly likely that even young children will be able to demonstrate them, at least in some rudimentary form. In this context, to avoid trying to "teach" our participants knowledge that they might have already mastered, we decided to use a new experimental procedure, composed of six phases - a pretest, four training phases, and a posttest - constructed to modify the traditional knowledge associated with the ELER heuristic and teach the children instead that Easily Learned $=$ Difficult to Remember.

As mentioned above, when a trials-to-acquisition paradigm is employed, the application of the ELER heuristic typically translates into higher JOLs for items that were learned after a small number of study trials than for those that were learned after a large number of trials, as measured by a JOL procedure. In the present study, this classical ELER effect was expected at pretest. However, because the participants were taught an opposite version of the ELER heuristic in the four training phases, we expected the pattern of JOL responses at posttest to be reduced or even reversed. Moreover, for the first time, we investigated the ELER heuristic not only in school-age children, but also in preschoolers. The finding that participants in the latter group use this heuristic would be a further indication of the involvement of early implicit processes in the learning of metamemory knowledge.

Finally, a secondary aim of this study was to explore the influence of three important executive functions - inhibition, flexibility, and executive monitoring — on the use of the ELER 
heuristic. In the literature, it has been argued that the mature implementation of a strategic heuristic requires inferential processes (Westerman, Miller, \& Lloyd, 2003) that could be subject to effortful regulation (e.g., Miller \& Lloyd, 2011). Therefore, once children attain a sufficient level of cognitive maturity, it is possible that they begin to apply effortful control to their learning and use of heuristics, thus becoming less sensitive to implicit environmental contingencies.

In sum, our aims for this study were (1) to determine whether young children are able to employ the ELER heuristic, (2) to examine the impact of implicit training on their application of this type of heuristic at posttest, and (3) to explore the influence of higher cognitive functions on their use of this type of heuristic.

\section{Method}

\subsection{Participants}

Participants were 60 typically developing children aged 4 to 5 (Mean $=60.64, \mathrm{SD}=$ 6.31), 6 to 7 (Mean $=81.77, \mathrm{SD}=7.76)$, and 8 to 9 years old $($ Mean $=108.29, \mathrm{SD}=5.73)$. $\mathrm{A}$ pretest carried out in a group of 12 participants was used to determine the sample size. Data collection stopped when the number of participants was sufficient to reach a predicted power of .80. The proportion of girls and boys was strictly equivalent in each group. No group difference was found in terms of parental education level and nonverbal intelligence, respectively assessed using both parents' years of education and scores on the Matrix Reasoning test (Wechsler, 2004, 2005). The sample was recruited from French-speaking kindergartens and elementary schools in the province of Liège, Belgium.

\subsection{Materials}

Stimuli consisted of six lists of 24 pairs of black-and-white pictures (cue-target) taken from the standardized sets developed by Cycowicz, Friedman, Rothstein, and Snodgrass (1997) and Bonin, Peereman, Malardier, Méot, and Chalard (2003). The pairs were matched so that no easy semantic or visual association could be made between the two pictures in a given pair (e.g., Bin-Lion). An additional set of 382 pictures was retrieved from the two databases to be used as lures on recognition tests.

\subsection{Procedure}

The study was approved by the local ethics committee. Written consent was obtained from the parents before the study began. Children were tested individually in a quiet room in their school, using a touchscreen computer. Each child participated in three 60-minute sessions approximately one week apart. Session 1 included the pretest and the first training phase, Session 2 consisted of two training phases, and Session 3 comprised the last training phase and the posttest. One of the six lists of stimuli was randomly assigned to each of the six experimental phases and counterbalanced between subjects. As Figure 1 shows, each experimental phase was composed of different steps: namely, (1) study-test loop, (2) JOL, and (3) recognition step.

Study-test loop. First, a list of 24 pairs of pictures was presented in random order $(3 \mathrm{~s}$ each) to each child. Participants were asked to try to remember as many pairs as possible in order to be able to recall as many targets as possible when the associated cues were presented later. Once every picture had been studied, a cued recall test was administered: the task was to recall the second picture of each pair in response to the presentation of the first one. At the end of the cued recall test, pairs that the participant failed to recall were presented for re-study and then 
tested again (Bahrick \& Phelps, 1987; Karpicke \& Roediger, 2008). This study-test loop ended when children attained perfect recall. At that point, for each participant, the picture pairs were sorted into three groups of equal size depending on the ease with which they had been learned (easy/medium/hard). Specifically, the items that were recalled earliest during the study-test loop were classified as "easy," while the items that were recalled latest were classified as "hard."

Judgment of learning (JOL). In the pre- and posttest phases, the first study step was immediately followed by a JOL solicitation. The pairs were presented again for $3 \mathrm{~s}$ and the participants were instructed to indicate the likelihood that they would recall the target picture on an upcoming cued-recall test. A thermometer procedure based on the hot/cold game (Koriat \& Shitzer-Reichert, 2002) was used to enable young children to understand the meaning of JOL. Specifically, a colored horizontal thermometer appeared on the computer screen with a cursor positioned in the middle. Children were asked to give their ratings by moving the cursor anywhere from the deep blue (very cold) end to the deep red (very hot) end of the scale according to their level of confidence. The position of the cursor on the thermometer was transformed into a JOL percentage score $(0 \%-100 \%)$.

Recognition. A 10-minute delay followed either the study period (in the training phases) or the JOL test (in the pre- and posttest phases). Once this time had elapsed, there was a final recognition test. Specifically, the children were required to choose which of four pictures was the target associated with the provided cue. In the baseline phases, the picture lures were (1) a target from another pair, (2) a semantic associate of the "true" target, and (3) an unrelated picture. However, in the four training phases, the recognition test was altered to forestall the application of the ELER heuristic, which is why a classical cued-recall task was not employed as in the previous stage (study-test loop) of the experimental procedure. Two concomitant actions were implemented to make recall of the easily learned pairs harder and facilitate recall of the harderto-learn pairs. First, the picture lures were "rigged" for easy and hard pairs: namely, targets of the easy pairs (e.g., Lion) were presented only with hard-to-reject lures-for example, (1) a semantic associate of the target (e.g., Tiger), (2) a target from another studied pair (e.g., Trumpet), and (3) a semantic associate of this "false" target (e.g., Saxophone) —whereas the lures that accompanied the targets in the hard pairs-three unrelated pictures-were chosen to be rejected with ease (e.g., Witch, Glasses, and Scaffolding). A priming procedure was also used to facilitate the processing of the hard pairs and make the processing of the easy pairs more difficult. Specifically, the targets of the hard pairs were presented for $17 \mathrm{~ms}$ just before the children had to identify them in the recognition test. Conversely, the false targets were primed just before the easy pairs were presented for recognition. Unrelated lures were primed before the recognition test for each medium-difficulty pair. Picture primes were directly followed by black masks that appeared for $17 \mathrm{~ms}$ before the recognition test.

During the 10-minute interval that preceded the recognition test, the participants were given nonverbal cognitive tasks such as the abstract self-ordered pointing test (SOPT) assessing the executive ability to generate and monitor a sequence of responses (Cragg \& Nation, 2007), a go/no-go test of response inhibition (Raaijmakers et al., 2008), the Dragons' House test of flexibility from the attentional test battery for children (KiTAP; Zimmermann, Gondan, \& Fimm, 2005), and the Matrix Reasoning test (Wechsler, 2004, 2005).

Finally, two problematic screenplays involving the ELER heuristic (e.g., "Would you find it easier to remember an easy song after listening to me sing it once or a more difficult song after listening to me sing it several times?") were presented to the children, who were then required to select one of two alternative responses (forced choice, for a maximum of two points). 
The aim of these two scenarios was to investigate explicit verbalization of the metacognitive heuristic. Consequently, the questions were constructed so as to require the children to consciously call upon their knowledge of the ELER rule in order to answer them. Afterward, the participants were asked whether they had noticed anything special about the test, and then were thanked.

\section{$<$ Figure $1>$}

\subsection{Measures}

The measures used in the analyses were (1) the mean of children's JOLs at pre- and posttest for the easy/medium/hard pairs, (2) the number of correct responses in the pretest and posttest phases, and (3) the number of errors produced on each of the three executive tasks.

\section{Results}

\subsection{Preliminary analyses}

Preliminary analyses indicated no order effect on any of the dependent variables. As can be seen in Table 1, results of the Kruskal-Wallis analyses revealed a significant main effect of age for the number of loops required to achieve perfect recall during the study phase at pre- and posttest. Similarly, a significant difference was highlighted between age groups for the three executive scores. However, no age effect was found on children's verbalization of the heuristic.

\section{$<$ Table $1>$}

\subsection{Pretest}

Our two primary goals for the pretest phase were (1) to determine whether childrenwith a particular focus on the youngest ones-are able to employ the ELER metacognitive heuristic, and (2) to ensure the relevance of this heuristic in guiding the JOL in our experiment by proving that the most easily learned items were in fact the most easily recognized. For this purpose, repeated measures planned comparisons were first conducted to assess the difference between JOLs for easy and hard pairs of pictures. As Figure 2 shows, the results of the linear contrast reveal that the mean JOL of the easy pairs was higher than the mean JOL of the hard pairs for the whole sample $\left(\mathrm{M}_{\mathrm{diff}}=15.04,95 \%\right.$ CI $\left.[9.84,20.26], p<.001, \eta_{\mathrm{p}}{ }^{2}=.36\right)$ and for 4year-olds $\left(\mathrm{M}_{\mathrm{diff}}=14.12,95 \% \mathrm{CI}[5.92,22.32], p=.002, \eta_{\mathrm{p}}{ }^{2}=.41\right), 6$-year-olds $\left(\mathrm{M}_{\mathrm{diff}}=15.62\right.$, $95 \%$ CI [5.76, 25.49], $\left.p=.004, \eta_{\mathrm{p}}{ }^{2}=.36\right)$, and 8-year-olds $\left(\mathrm{M}_{\text {diff }}=15.39,95 \%\right.$ CI [4.80, 25.97], $\left.p=.007, \eta_{\mathrm{p}}^{2}=.33\right)$. It is also interesting to note that the number of children who exhibited the expected ELER heuristic pattern of responses (JOL for easy pairs > JOL for hard pairs) was strictly equivalent in each age group $(n=16)$. Second, to show that the ELER heuristic is truly an appropriate strategy to guide memory predictions, another linear contrast was carried out. The results indicated a moderately sized difference between the number of easy pairs and the number of hard pairs that children correctly identified during the recognition test $\left(\mathrm{M}_{\mathrm{diff}}=0.3,95 \% \mathrm{CI}\right.$ $\left.[0.02,0.57], p=.03, \eta_{\mathrm{p}}^{2}=.08\right)$, thus demonstrating the appropriateness of the use of the ELER heuristic in the present study.

In a more exploratory spirit, the influence of some higher cognitive functions on children's use of the ELER heuristic was also investigated. Here, participants' use of the ELER heuristic was assessed by subtracting the mean JOL for hard pairs from the mean JOL for easy pairs, with a positive score indicating greater confidence for easily encoded information than for 
information that was hard to encode. The results of the multiple linear regression revealed that none of the three executive variables included in the analysis could satisfactorily explain the children's use of the ELER heuristic, either for the whole sample $(p=.61)$ or for any of the three age groups ( $p=.85, .23$, and .16 for the 4-, 6-, and 8-year-old groups, respectively).

\section{$<$ Figure 2 >}

\subsection{Posttest}

As illustrated in Figure 2, the use of the ELER heuristic was mostly reduced at posttest compared to what was observed at pretest. Specifically, the comparison of the difference between mean JOLs for easy and hard pairs revealed a substantial decrease in the use of the ELER heuristic between the pretest and posttest phases in the whole sample $\left(\mathrm{M}_{\text {diff }}=12.83,95 \%\right.$ CI [6.69, 18.97], $\left.p<.001, \eta_{\mathrm{p}}{ }^{2}=.23\right)$, in 4-year-olds ( $\mathrm{M}_{\mathrm{diff}}=20.61,95 \%$ CI [8.48, 32.74], $p=$ $\left..002, \eta_{\mathrm{p}}{ }^{2}=.40\right)$, and in 6-year-olds $\left(\mathrm{M}_{\mathrm{diff}}=10.75,95 \% \mathrm{CI}[0.33,21.17], p=.04, \eta_{\mathrm{p}}{ }^{2}=.20\right)$. Children in the oldest group, however, seemed to be less affected by the experimental procedure $\left(\mathrm{M}_{\text {diff }}=7.14,95 \%\right.$ CI $\left.[-3.17,17.44], p=.16, \eta_{\mathrm{p}}{ }^{2}=.10\right)$. Nevertheless, children still performed better on the recognition test for easy than for hard pairs $\left(\mathrm{M}_{\mathrm{diff}}=0.27,95 \%\right.$ CI [0.01, 0.53], $p=$ $\left..03, \eta_{\mathrm{p}}{ }^{2}=.07\right)$, proving that, even though some participants had learned to cease using it, the ELER heuristic remained an appropriate way to predict future memory performance.

Finally, a multiple regression analysis was carried out to explore the possible influence of executive functions on children's pattern of JOLs after the four learning phases. The difference between posttest JOLs for easy and hard pairs was used as dependent variable. As Table 2 shows, executive monitoring - assessed by the number of errors made on the SOPT-predicted the oldest children's use of the ELER heuristic at posttest $\left(\beta=-.55, p=.047, \mathrm{R}^{2}=.23\right)$. More specifically, in the 8-year-old group, the children with the best monitoring abilities were also the ones who most resisted the experimental manipulation and whose JOLs were thus relatively unchanged at posttest compared to at pretest. None of the three executive variables contributed to explaining the JOLs of 4-year-olds and 6-year-olds at posttest.

$<$ Table $2>$

\section{Discussion}

The primary goal of this study was to highlight the mechanisms involved in the learning of metacognitive heuristics. In this experiment, a number of interesting results were obtained which provide further information on the experiential explanation of the development of metamemory knowledge (Jacoby et al., 1989; Olds \& Westerman, 2012) and suggest some promising potential pathways to explain how these heuristics are acquired during childhood.

One of these results concerns the ability of the preschool-age group to use the ELER heuristic in the first baseline phase. The difference between this group's JOLs for easy and hard pairs at pretest indicates that children of this age are able to base their memory predictions on the knowledge that easily encoded information is more likely to be remembered. Combined with the fact that no executive variable included in the analyses could satisfactorily explain the use of the ELER heuristic at pretest, this finding seems to suggest that — unlike what is observed for explicit metamemory knowledge (Grammer et al., 2011) — metacognitive heuristics are not learned through late and effortful processes, but rather through earlier and more automatic ones. 
The changes in JOLs between pre- and posttest observed in the 4-year-old and 6-year-old groups seem to confirm this view. Unlike Unkelbach's (2006) observations with the fluency heuristic, our results do not show a complete reversal of the ELER effect. It seems that perceptual fluency can be modulated more directly (i.e., systematic association of new stimuli with high perceptual fluency) than ease of remembering. This is presumably why the actions that we took to disrupt the relationship between ease of learning and ease of remembering did not result in a correlation as perfect as the one created by Unkelbach. Nevertheless, a significant decrease in the participants' reliance on the heuristic was still demonstrated. Specifically, our findings indicate that the children in the two youngest groups modified their JOLs according to the new metacognitive contingencies that they were exposed to in the training phases, proving that they were able to implicitly detect metamemorial regularities and use them to guide their subsequent behavior. In contrast, the 8-year-old children appear to have been less sensitive to the learning procedure even though, like the children in the other age groups, none of them reported having noticed either of the two experimental manipulations implemented to thwart the use of the ELER heuristic. But given that the ability to detect environmental regularities had already developed in the younger children, what might explain the apparent absence of implicit learning in the oldest group?

Several potential explanations can be posited. One involves the possibility that the ELER heuristic may be more firmly established, and thus less malleable, in older children. However, since the number of children who used the ELER heuristic at pretest was equivalent in each group ( $\mathrm{n}=16$ ), as were the effect sizes indicating the strength of the heuristic (from .41 to .33), this hypothesis should probably be rejected. These two results suggest that the children in the three age groups mastered the ELER rule to a similar extent.

A second hypothesis that could explain the apparent lack of implicit learning in 8-yearold children could reside in contamination by explicit knowledge. Children with verbally accessible knowledge of the ELER rule might have based their JOLs on it regardless of what they implicitly perceived in the training phases. Nonetheless, in addition to the fact that the ELER heuristic was specially chosen because people have been found to have no explicit knowledge of it (Koriat \& Ackerman, 2010), the analysis carried out on scores for explicit verbalization of the heuristic did not reveal any difference between age groups. In other words, children in the oldest group did not express more explicit knowledge of the heuristic than the younger children.

However, the failure of the experimental paradigm to affect the performance of 8-yearold children could also be explained by their better performance in the study-test loops. For example, imagine a child who had retrieved the 24 picture pairs within a single iteration of the series. This child would most probably not be able to learn the new metacognitive rule: the detection of the new rule requires subjects to distinguish between pairs of pictures according to the level of difficulty involved in learning them, but for this child, all pairs would have been equally difficult. Less generally, it might be thought that the oldest children's failure to learn the reverse metacognitive heuristic resulted from a loss of discriminability due to the small number of trials that these children needed to achieve perfect recall. Once again, however, other findings lead us to reject this proposal. Participants in the oldest group were able to distinguish between easy and hard pairs, as shown by their demonstration of the classical ELER heuristic pattern of responses at pretest. Furthermore, the number of trials required to learn the picture lists remained stable across the six test phases $(\mathrm{p}=.62)$. If a given number of trials was sufficient to 
differentiate easy pairs from hard ones at pretest, a similar number of trials should be adequate to derive the same distinction in the training and posttest phases as well.

A final possibility is Miller and Lloyd's (2011) hypothesis that the learning and use of heuristics can be controlled through effortful processes once children achieve a sufficient level of cognitive maturity. The results of the regression analyses seem to confirm this assumption: in the 8-year-old group, the children with the best executive monitoring skills showed less implicit learning, which may suggest that, at some point in their development, children start to use effortful processes to monitor and, probably, support the learning of metacognitive heuristics.

\section{Conclusion}

This study has suggested that the ELER heuristic appears to be learned very early, and has investigated the processes underlying this learning. The results have not only provided evidence that some basic memory association rules could be developed through an implicit process involving the detection of environmental regularities, they also seem to support Miller and Lloyd's hypothesis that, once they have matured sufficiently, high-level cognitive functions may regulate the use of these heuristics. These findings will, of course, need to be corroborated and generalized to other metacognitive heuristics and other sorts of memory tests. Another study recently carried out by our research unit using the distinctiveness heuristic produced results that support the present findings, and a paper is currently in preparation to present those results. Nonetheless, the present findings seem, for the first time, to shed light, consistently and in detail, on the experiential explanation of the development of metacognitive heuristics. 
Acknowledgements

This research was supported by the Belgian Fund for Scientific Research (F.R.S.-FNRS). 


\section{References}

Bahrick, H. P., \& Phelps, E. (1987). Retention of Spanish vocabulary over 8 years. Journal of Experimental Psychology: Learning, Memory, and Cognition, 13, 344-349. doi:10.1037/0278-7393.13.2.344

Bonin, P., Peereman, R., Malardier, N., Méot, A., \& Chalard, M. (2003). A new set of 299 pictures for psycholinguistic studies: French norms for name agreement, image agreement, conceptual familiarity, visual complexity, image variability, age of acquisition, and naming latencies. Behavior Research Methods, Instruments, and Computers, 35, 158-167.

Cragg, L., \& Nation, K. (2007). Self-ordered pointing as a test of working memory in typically developing children. Memory, 15, 526-535.

Cycowicz, Y. M., Friedman, D., Rothstein, M., \& Snodgrass, J. G. (1997). Picture naming by young children: Norms for name agreement, familiarity, and visual complexity. Journal of Experimental Child Psychology, 65, 171-237.

Dodson, C., \& Schacter, D. (2001). "If I had said it I would have remembered it": Reducing false memories with a distinctiveness heuristic. Psychonomic Bulletin and Review, 8, 155-161.

Ghetti, S. (2008). Rejection of false events in childhood: A metamemory account. Current Directions in Psychological Science, 17, 16-20. doi:10.1111/j.1467-8721.2008.00540.x

Gomez, R. L., \& Gerken, L. (1999). Artificial grammar learning by 1-year-olds leads to specific and abstract knowledge. Cognition, 70, 109-135.

Grammer, J. K., Purtell, K. M., Coffman, J. L., \& Ornstein, P. A. (2011). Relations between children's metamemory and strategic performance: Time-varying covariates in early elementary school. Journal of Experimental Child Psychology, 108, 139-155.

Hodzik, S., \& Lemaire, P. (2011). Inhibition and shifting capacities mediate adults' age-related differences in strategy selection and repertoire. Acta Psychologica, 137, 335-344.

Howe, M. L. (2008). Visual distinctiveness and the development of children's false memories. Child Development, 79, 65-79.

Jacoby, L. L., Kelley, C. M., \& Dywan, J. (1989). Memory attributions. In H. L. Roediger III \& F. I. M. Craik (Eds.), Varieties of memory and consciousness: Essays in honour of Endel Tulving (pp. 391-422). Hillsdale, NJ: Lawrence Erlbaum Associates, Inc.

Johnston, W. A., Hawley, K. J., \& Elliott, J. M. (1991). Contribution of perceptual fluency to recognition judgments. Journal of Experimental Psychology: Learning, Memory, and Cognition, 17, 210-223.

Karpicke, J. D., \& Roediger, H. L. (2008). The critical importance of retrieval for learning. Science, 319, 966-968.

Koriat, A. (2008). Easy comes, easy goes? The link between learning and remembering and its exploitation in metacognition. Memory and Cognition, 36, 416-428.

Koriat, A., \& Ackerman, R. (2010). Metacognition and mindreading: Judgments of learning for self and other during self-paced study. Consciousness and Cognition, 19, 251-264.

Koriat, A., Ackerman, R., Adiv, S., Lockl, K., \& Schneider, W. (2014). The effects of goaldriven and data-driven regulation on metacognitive monitoring during learning: A developmental perspective. Journal of Experimental Psychology: General, 143, 386-403.

Koriat, A., Ackerman, R., Lockl, K., \& Schneider, W. (2009a). The easily learned, easily remembered heuristic in children. Cognitive Development, 24, 169-182. 
Koriat, A., Ackerman, R., Lockl, K., \& Schneider, W. (2009b). The memorizing effort heuristic in judgments of learning: A developmental perspective. Journal of Experimental Child Psychology, 102, 265-279.

Koriat, A., Ma'ayan, H., \& Nussinson, R. (2006). The intricate relationships between monitoring and control in metacognition: Lessons for the cause-and-effect relation between subjective experience and behavior. Journal of Experimental Psychology: General, 135, 36-69.

Koriat, A., Nussinson, R., \& Ackerman, R. (2014). Judgments of learning depend on how learners interpret study effort. Journal of Experimental Psychology: Learning, Memory, and Cognition. Advance online publication.

Koriat, A., \& Shitzer-Reichert, R. (2002). Metacognitive judgments and their accuracy. In P. Chambres, M. Izaute \& P.-J. Marescaux (Eds.), Metacognition: Process, function and use (pp. 1-17). New York: Springer.

Lockl, K., \& Schneider, W. (2002). Developmental trends in children's feeling-of-knowing judgements. International Journal of Behavioral Development, 26, 327-333.

Lyons, K. E., \& Ghetti, S. (2013). I don't want to pick! Introspection on uncertainty supports early strategic behavior. Child Development, 84, 726-736.

Meeks, J. T., Knight, J. B., Brewer, G. A., Cook, G. I., \& Marsh, R. L. (2014). Investigating the subjective reports of rejection processes in the word frequency mirror effect. Consciousness and Cognition, 24, 57-69.

Meulemans, T., Van der Linden, M., \& Perruchet, P. (1998). Implicit sequence learning in children. Journal of Experimental Child Psychology, 69, 199-221.

Miele, D. B., Finn, B., \& Molden, D. C. (2011). Does easily learned mean easily remembered? It depends on your beliefs about intelligence. Psychological Science, 22, 320-324.

Miller, J. K., \& Lloyd, M. E. (2011). The development of the fluency heuristic in childhood: More questions than answers. In P. A. Higham \& J. P. Leboe (Eds.), Constructions of remembering and metacognition (pp. 28-39). Basingstoke, UK: Palgrave Macmillan.

Olds, J. M., \& Westerman, D. L. (2012). Can fluency be interpreted as novelty? Retraining the interpretation of fluency in recognition memory. Journal of Experimental Psychology: Learning, Memory, and Cognition, 38, 653-664.

Paulus, M., Proust, J., \& Sodian, B. (2013). Examining implicit metacognition in 3.5-year-old children: An eye-tracking and pupillometric study. Frontiers in Psychology, 4, 1-7.

Paulus, M., Tsalas, N., Proust, J., \& Sodian, B. (2014). Metacognitive monitoring of oneself and others: Developmental changes during childhood and adolescence. Journal of Experimental Child Psychology, 122, 153-165.

Raaijmakers, M. A., Smidts, D. P., Sergeant, J. A., Maassen, G. H., Posthumus, J. A., van Engeland, H., \& Matthys, W. (2008). Executive functions in preschool children with aggressive behavior: Impairments in inhibitory control. Journal of Abnormal Child Psychology, 36, 1097-1107.

Roebers, C. M., von der Linden, N., Schneider, W., \& Howie, P. (2007). Children's metamemorial judgments in an event recall task. Journal of Experimental Child Psychology, 97, 117-137.

Tversky, A., \& Kahneman, D. (1973). Availability: A heuristic for judging frequency and probability. Cognitive Psychology, 5, 207-232. 
Undorf, M., \& Erdfelder, E. (2011). Judgments of learning reflect encoding fluency: Conclusive evidence for the ease-of-processing hypothesis. Journal of Experimental Psychology: Learning, Memory, and Cognition, 37, 1264-1269.

Unkelbach, C. (2006). The learned interpretation of cognitive fluency. Psychological Science, $17,339-345$.

Unkelbach, C. (2007). Reversing the truth effect: Learning the interpretation of processing fluency in judgments of truth. Journal of Experimental Psychology: Learning, Memory, and Cognition, 33, 219-230.

Wechsler, D. (2004). Echelle d'intelligence de Wechsler pour la période préscolaire et primaire: WPPSI-III. Paris: Editions du Centre de Psychologie Appliquée.

Wechsler, D. (2005). Echelle d'intelligence de Wechsler pour enfants: WISC-IV. Paris: Les Editions du Centre de Psychologie Appliquée.

Westerman, D., Miller, J., \& Lloyd, M. (2003). Change in perceptual form attenuates the use of the fluency heuristic in recognition. Memory and Cognition, 31, 619-629.

Winkielman, P., \& Schwarz, N. (2001). How pleasant was your childhood? Beliefs about memory shape inferences from experienced difficulty of recall. Psychological Science, $12,176-179$.

Zimmermann, P., Gondan, M., \& Fimm, B. (2005). KiTAP. Test d'évaluation de l'attention. Version pour enfants. Herzogenrath, Germany: Psytest. 